\title{
Die Autoren dieser Sonderausgabe stellen sich vor, mit herzlichem Dank an Prof. Storch
}

\author{
Henner Hollert • Thomas Braunbeck (Herausgeber)
}

Eingegangen: 23. Februar 2009/Akzeptiert: 1. März 2009/Online veröffentlicht: 20. März 2009

(C) Springer-Verlag 2009

\section{Morphologische Parameter als Biomarker in der Ökotoxikologie - natürliche und schadstoffinduzierte Variabilität}

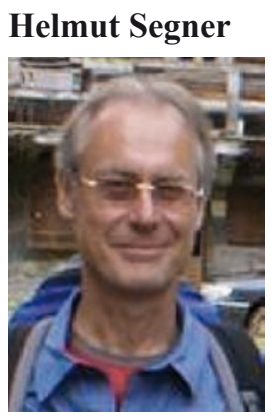

Prof. Helmut Segner leitet das Zentrum für Fisch- und Wildtiermedizin an der Vetsuissse Fakultät der Universität Bern. Nach der Promotion am Institut für Zoomorphologie der Universität Heidelberg war er als Hochschulassistent am Institut für Zoophysiologie der Universität Karlsruhe sowie als Leiter der Arbeitsgruppe Aquatische Ökotoxikologie am Umweltforschungszentrum Leipzig tätig, bevor er im Jahr 2000 nach Bern wechselte. Seine Forschungsinteressen gelten den physiologischen und pathologischen Reaktionen von Fischen auf Belastung mit Umweltstressoren, insbesondere toxische Chemikalien und Pathogene.

Dieser Beitrag ist Herrn Prof. Dr. Dr. h.c. Volker Storch zum 65. Geburtstag gewidmet.

H. Hollert $(\bowtie)$

Lehr- und Forschungsgebiet für Ökosystemanalyse,

Institut für Umweltforschung (Biologie V),

Rheinisch-Westfälische Technische Hochschule Aachen,

Worringerweg 1, 52074 Aachen, Deutschland

E-Mail: henner.hollert@bio5.rwth-aachen.de

T. Braunbeck

Aquatische Ökologie und Toxikologie, Institut für Zoologie, Universität Heidelberg,

Im Neuenheimer Feld 230, 69120 Heidelberg, Deutschland

E-Mail: braunbeck@zoo.uni-heidelberg.de

\author{
Schnecken unter Stress \\ Gastropoden als Modelle in Ökophysiologie \\ und Ökotoxikologie
}

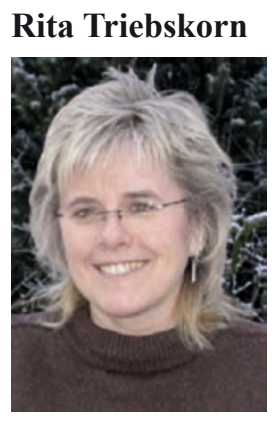

Prof. Dr. Rita Triebskorn ist Forschungsgruppenleiterin in der Abteilung Physiologische Ökologie der Tiere der Universität Tübingen und seit neun Jahren Leiterin des SteinbeisTransferzentrums für Ökotoxikologie und Ökophysiologie, Rottenburg. Ihr wissenschaftliches Interesse gilt einerseits den Wirkungen von Umweltchemikalien v. a. im Zusammenspiel mit anderen Umweltfaktoren bei wirbellosen Tieren (Schwerpunkt Mollusken) und Fischen. Darüber hinaus beschäftigt sie sich mit Studien zu Wirkmechanismen von Molluskiziden, die im Zusammenhang mit der Effizienzsteigerung sowie einer verbesserten Umweltverträglichkeit der jeweiligen Produkte stehen.

Rita Triebskorn studierte von 1980-1987 Biologie und Germanistik für das Höhere Lehramt an Gymnasien und promovierte 1990 am Zoologischen Institut I (Morphologie/ Ökologie) der Universität Heidelberg unter der Betreuung von Herrn Prof. Dr. Volker Storch. 
Die Rolle von Stressproteinen bei der Anpassung an Umweltbedingungen: Ökophysiologische, ökotoxikologische und evolutionsbiologische Implikationen

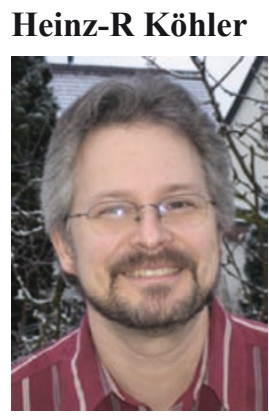

Prof. Dr. Heinz-R. Köhler leitet seit neun Jahren die Abteilung Physiologische Ökologie der Tiere und ist seit 2009 Direktor des neu gegründeten Instituts für Evolution und Ökologie an der Universität Tübingen. Er studierte Biologie an der Universität Heidelberg und fertigte sowohl seine Diplomarbeit als auch seine Dissertation 1992 unter Betreuung von Prof. Dr. Gerd Alberti im von Prof. Dr. Volker Storch geleiteten Zoologischen Institut I (Morphologie/ Ökologie) an. Sein wissenschaftliches Interesse gilt insbesondere stressphysiologischen und evolutionsbiologischen Aspekten der Ökophysiologie und Ökotoxikologie von wirbellosen Tieren und Fischen.

\section{Strategien zur Sedimentbewertung - ein Überblick}

\section{Henner Hollert • Marit Ernst • Wolfgang Ahlf • Matthias Dürr · Lothar Erdinger · Stefanie Grund • Steffen Keiter - Thomas Kosmehl - Thomas-Benjamin Seiler · Jan Wölz $\cdot$ Thomas Braunbeck}

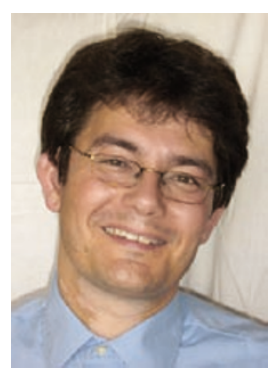

Prof. Dr. Henner Hollert leitet seit Oktober 2007 das Lehr- und Forschungsgebiet Ökosystemanalyse am Institut für Umweltforschung der RWTH Aachen. Von 2001 bis 2007 war er Arbeitsgruppenleiter in der Sektion Aquatische Toxikologie \& Ökologie (Prof. Dr. Thomas Braunbeck) und Wissenschaftlicher Assistent von Herrn Prof. Storch am Zoologischen Institut der Universität Heidelberg. Er studierte Biologie und Geografie an der Universität Heidelberg und fertigte sowohl seine Diplomarbeit als auch seine Dissertation am Zoologischen Institut an. Er beschäftigt sich mit zellbasierter wirkungsorientierter Analytik und der ökotoxikologischen Gefährdungsabschätzung in aquatischen und terrestrischen Systemen. Schwerpunkte sind Sedimenttoxikologie, die ökologische Systemanalyse mittels Weight-of-Evidence-Ansätzen, Hochwasserfolgenbewertung, der ökotoxikologischen Bewertung von Biofuels, Bioassay-dirigierten Fraktionierungen sowie die Bewertung komplexer Datenmatrices. Seit 2005 ist er Herausgeber von UWSF - Z Umweltchem Ökotox und in den Herausgebergremien von Environ Sci Pollut Res (ESPR) und J Soils Sediments (JSS) als Subject-Editor tätig.

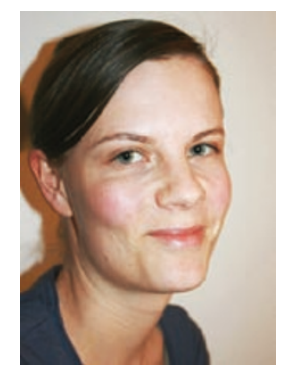

Marit Ernst ist Diplom-Biologin und derzeit am Lehr- und Forschungsgebiet Ökosystemanalyse der RWTH Aachen tätig. Schwerpunkte ihres Studiums an der Universität Heidelberg waren Zoologie, Ökologie sowie Geografie. Auch in ihrer Diplomarbeit, in der sie mithilfe von Biotestmethoden die endokrine Belastung von Sedimenten des Skadarsees in Montenegro untersuchte, konnte sie durch den Aufbau eines Geografischen Informationssystems (GIS) biologische Fragestellungen mit geografischen Aspekten verbinden.

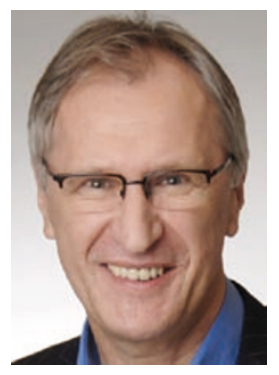

PD Dr. habil. Wolfgang Ahlf leitet eine ökotoxikologische Arbeitsgruppe an der TU Hamburg-Harburg und betreibt dort seit über 25 Jahren Forschung und Lehre. Seine Forschungsinteressen sind biologische Testverfahren, mit denen die Wirkungen partikelgebundener Schadstoffe nachzuweisen sind. Das Untersuchungsspektrum umfasst sowohl limnische als auch marine Sedimente, Böden und zunehmend Feinstäube aus Verbrennungsprozessen. Ein eng mit der Anwendung von Biotests verbundenes Tätigkeitsfeld ist die Interpretation und Bewertung der Daten. Die Anwendung multivariater Statistik zur Interpretation komplexer Datensätze bis hin zur Modelbildung (Risk Assessment) für eine Sedimentbewertung nimmt nicht nur wissenschaftlich einen beträchtlichen Raum ein, sondern führte auch zu festen Kooperationen mit Bundes- und Landesbehörden.

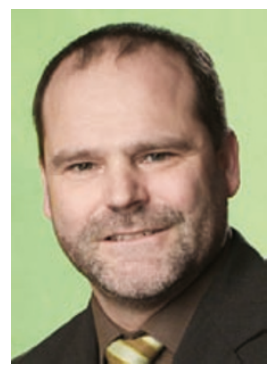

Dr. Matthias Dürr ist wissenschaftlicher Mitarbeiter im Institut für Hygiene der Martin-Luther-Universität HalleWittenberg. Er beschäftigt sich mit wirkungsorientierter Analytik mithilfe von chemischen sowie Bioassay-basierten Techniken, insbesondere von gentoxischen bzw. mutagenen Schadstoffen. Ein weiteres Themenfeld besteht in der Keimreduktion von Trink-, Brauch- und Abwässern.

Schwerpunkte sind Hochwasseranalysen, Bioassay-dirigierte Fraktionierungen von komplexen Schadstoff-Matrices wie remobilisierten Altsedimenten und partikelgebunden Luftschadstoffen, Keimreduktion durch naturnahe Abwasserreinigung und Keimreduktion von Betriebswässern in Medizinprodukten.

Er ist Subject Editor der UWSF - Z Umweltchem Ökotox und hier verantwortlich für Umwelthygiene und Umweltmedizin. 


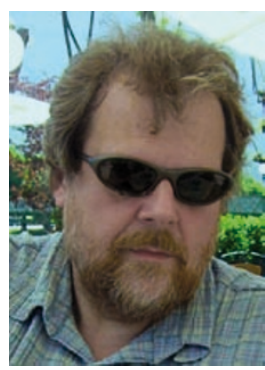

Priv.-Doz. Dr. Lothar Erdinger beschäftigt sich seit 1984 mit der Bildung von Desinfektionsnebenprodukten bei der Aufbereitung von Schwimm- und Badebeckenwasser. Er ist stellvertretender Vorsitzender der Schwimm- und Badebeckenwasserkommission des Bundesministeriums für Gesundheit am Umweltbundesamt. Schwerpunkt der Arbeitsgruppe am Hygiene Institut der Universität Heidelberg, die Herr Erdinger leitet, ist die Identifizierung mutagener und endokriner Einzelverbindungen aus komplexen Stoffgemischen. Seit einigen Jahren werden daneben Arbeiten zum photokatalytischen Abbau von Schadstoffen auf Oberflächen durchgeführt. Lothar Erdinger ist Mitglied des Editorial Board von ESPR und von Chemosphere.

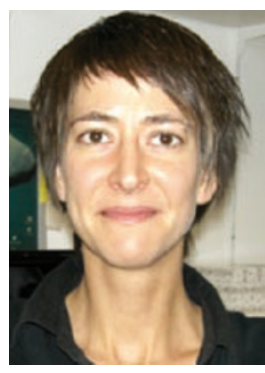

Stefanie Grund arbeitet seit 2004 in der Sektion Aquatische Toxikologie und Ökologie am Institut für Zoologie der Universität Heidelberg. Im Rahmen ihrer Diplom- und Doktorarbeit führte sie in der AG Braunbeck/Hollert im Sinne einer Weight-of-Evidence-Studie verschieden ökotoxikologische in vitro- und in situ- Untersuchungen sowie

Bioassay-dirigierte Fraktionierungen durch, um potenzielle Ursachen für den Fischrückgang in der Oberen Donau zu identifizieren.

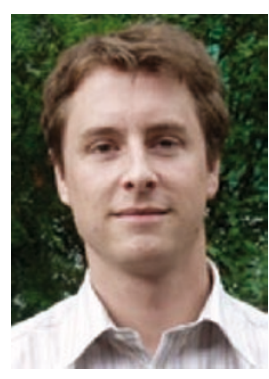

Dr. Thomas Kosmehl arbeitet seit Juli 2007 als regulatorischer Ökotoxikologe in der Abteilung Produktsicherheit Industriechemikalien der BASF SE.

Von 1997 bis 2003 studierte er Biologie an der Ruprecht-Karls-Universität in Heidelberg mit dem Hauptfach Ökologie. Bereits vor seinem Diplom begann er seine wissenschaftliche Mitarbeit in der Arbeitsgruppe „Aquatische Toxikologie und Ökologie“ von Prof. Thomas Braunbeck und Sedimenttoxikologie von Prof. Henner Hollert am Zoologischen Institut. Nach dem Diplom 2003 promovierte er in derselben Arbeitsgruppe über molekulare Biomarker an frühen Embryonalstadien des Zebrafisches. Im Rahmen der Dissertation entstanden u.a. Kooperationsprojekte mit der Bundesanstalt für Gewässerkunde, der Universität Tübingen und der University of Manila zur Bestimmung des toxischen Potenzials von Sedimentproben sowie mit dem Forschungszentrum Karlsruhe zur Untersuchung von Genexpressionsmustern in sedimentexponierten Zebrafischembryonen.

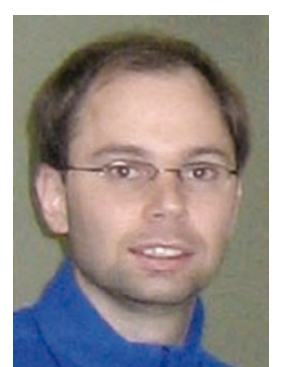

Thomas-Benjamin Seiler ist DiplomBiologe mit Heidelberger Ausbildung. Er arbeitete in seiner Diplomarbeit über Membrandialyse bei der Sedimentextraktion (AG Braunbeck/Hollert) und promoviert seit November 2004 über Extraktionsmethoden bei Prof. Dr. Thomas Braunbeck. Im Oktober 2007 ging er als wissenschaftlicher Assistent des Lehr- und Forschungsgebietes „Ökosystemanalyse“ von Prof. Dr. Henner Hollert nach Aachen. Er leitet dort die Forschungsgruppe „Effektbezogene Bioanalytik“, die mittels anwendungsorientierter Grundlagenforschung in den Bereichen Probenaufbereitung und Bioassays mit dem Ziel arbeitet, ökotoxikologische Untersuchungen einfacher und zugleich verlässlicher zu machen.

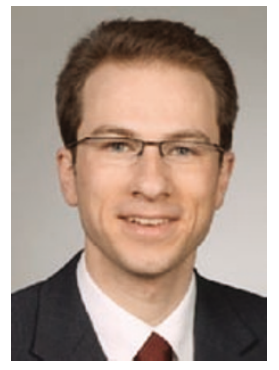

Dipl.-Biol. Jan Wölz promoviert derzeit in einem interdisziplinären Verbundprojekt (RIMAX-HoT) über das Schädigungspotenzial von (extremen) Hochwasserereignissen hinsichtlich der Belastung von Retentionsflächen sowie des Aquifers. Zunächst studierte er an der Ruprecht-Karls Universität in Heidelberg Biologie, an der er auch seine Diplomarbeit schrieb (AG Braunbeck/Hollert) und seine Promotion begann. Seit Ende 2007 führt er seine Promotion an der RWTH in Aachen fort und leitet am Lehr- und Forschungsgebiet Ökosystemanalyse die Arbeitsgruppe „Hochwasserfolgenbewertung“. Parallel zu seiner Promotion absolviert er den Postgradualstudiengang „Fachökotoxikologie“ von GDCh und SETAC. Seit 2003 ist er Mitglied der SETAC und nahm seither regelmäßig an den (inter-)nationalen Tagungen (GLB und EU), sowie an der SETAC-World-Tagung in Sydney teil. Zudem arbeitet er im AK Gentoxizität des DIN AK an der ISO-Standardisierung des Ames Fluktuationstests mit.

Klimawandel und Bachforellenrückgang - gibt es einen Zusammenhang? Resultate aus der Schweiz

\section{Patricia Burkhardt-Holm}

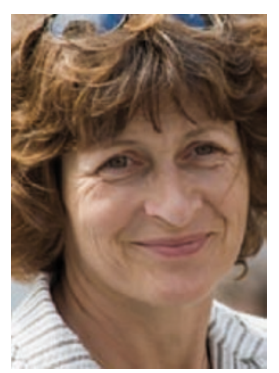

Prof. Dr. Patricia Burkhardt-Holm ist seit fünf Jahren Leiterin des Programms Mensch-Gesellschaft-Umwelt an der Universität Basel. Sie forscht im Bereich der aquatischen Ökologie und Fischbiologie. Im Fokus steht der Einfluss von natürlichen und anthropogenen Stressfaktoren und ihrer kumulativen Effekte. In den vergangenen Jahren hat sie durch inter- und transdisziplinäre Arbeiten zur Erfor- 
schung der Ursachen des Fischrückgangs in der Schweiz beigetragen und an der Bedeutung des Einflusses von endokrinen Disruptoren, der Temperatur, der Ökotoxikologie und von Feinsedimenten auf Fische gearbeitet. Sie leitet den trifakultären Masterstudiengang Sustainable Development. Ihr Biologie-Studium und ihre Doktorarbeit über den Darmkanal von Fischen absolvierte sie am Zoologischen Institut Heidelberg unter Leitung von Prof. Dr. Volker Storch, mit dem sie seitdem zusammenarbeitet und freundschaftlich verbunden ist.

\section{Der Fischrückgang in der oberen Donau}

\section{Steffen Keiter · Melanie Böttcher · Steffi Grund · Nadja Seitz · Thomas Braunbeck · Henner Hollert}

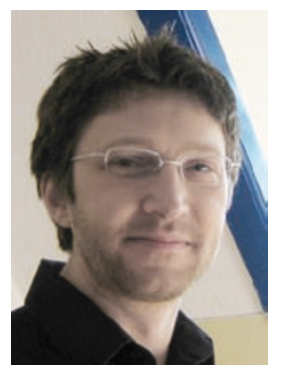

Dr. Steffen Keiter arbeitet seit Mitte 2008 als Projektmanager für Produktsicherheit bei der Dr. Knoell Consult $\mathrm{GmbH}$ in Mannheim. Zuvor war er in der Arbeitsgruppe Aquatische Toxikologie und Ökologie am Institut für Zoologie der Universität Heidelberg tätig. Schwerpunkte seiner Arbeit waren dort die Durchführung einer Weight-of-Evidence-Studie, um den Fischrückgang in der Oberen Donau zu klären und die Klassifizierung von Ergebnissen aus Sedimentbiotests in integrativen Bewertungskonzepten.

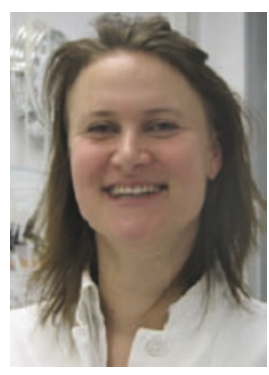

Dipl. Biol. Melanie Böttcher absolvierte ihr Studium der Biologie an der Universität Heidelberg. Seit August 2005 arbeitet sie in der Arbeitsgruppe Aquatische Toxikologie und Ökologie (Prof. Dr. Thomas Braunbeck) am Zoologischen Institut der Universität Heidelberg an ihrer Doktorarbeit mit dem Forschungsschwerpunkt endokrine Disruptoren und deren gentoxisches Potenzial. Des Weiteren arbeitet sie seit Februar 2008 bei der Ibacon GmbH in Roßdorf als Studienleiterin mit dem Zuständigkeitsbereich aquatische Toxikologie.

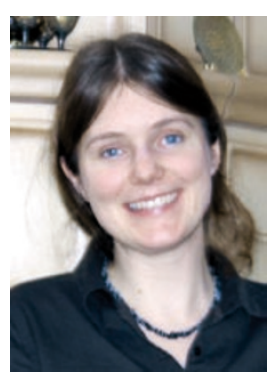

Dipl. Biol. Nadja Seitz arbeitet seit 2004 in der Arbeitsgruppe Aquatische Toxikologie und Ökologie am Institut für Zoologie der Universität Heidelberg. Schwerpunkt ihrer Arbeit ist die Untersuchung und Modellierung des intrazellulären Calciumspiegels in Primärhepatocyten der Regenbogenforelle.
Fischpopulationen unter Stress - das Beispiel des Unteren Neckars

\section{Thomas Braunbeck • Henner Hollert • Annika Brauns • Steffen Keiter · Patrick Schwartz}

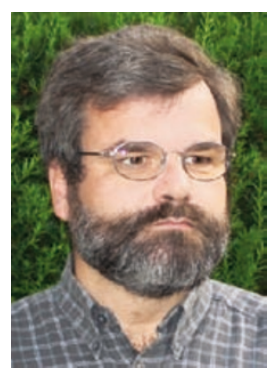

Prof. Dr. Thomas Braunbeck leitet die Arbeitsgruppe Aquatische Ökologie und Toxikologie am Institut für Zoologie der Universität Heidelberg. Die Forschungsschwerpunkte im Bereich der Aquatischen Ökotoxikologie liegen auf dem Nachweis gentoxischer, endokriner und histologischer sowie zellulärer Wirkungen bei Fischen. Ein weiterer Schwerpunkt liegt seit vielen Jahren auf der Entwicklung von Alternativmethoden zu Tierversuchen, wobei Arbeiten mit primären und permanenten Zellkulturen sowie Fischembryonen im Vordergrund stehen. In der Lehre vertritt er sowohl in Bachelor- als auch Master-Studiengängen neben der Ökotoxikologie vor allem die klassische Zoologie und Ökologie. Mitglied in zahlreichen ökotoxikologischen, ökologischen und zoologischen Fachgesellschaften, Vertreter in mehreren OECD-Gremien und Mitherausgeber mehrerer Zeitschriften.

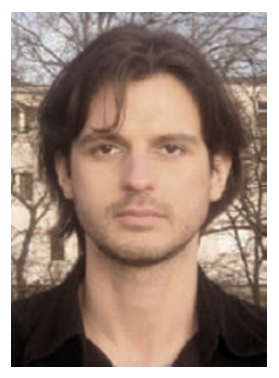

Dipl. Biologe Patrick Schwartz arbeitet seit Januar 2006 beim Programm Mensch-Gesellschaft-Umwelt an der Universität Basel und untersucht dort im Rahmen seiner Dissertation die Effekte von endokrinen Disruptoren und Mykotoxinen auf Fische. Zuvor studierte er Biologie an der Universität Karlsruhe und der Ruprecht-KarlsUniversität Heidelberg, wo er in der Arbeitsgruppe Aquatische Ökologie und Toxikologie am Institut für Zoologie auch seine Diplomarbeit über die Fischfauna im Unteren Neckar anfertigte.

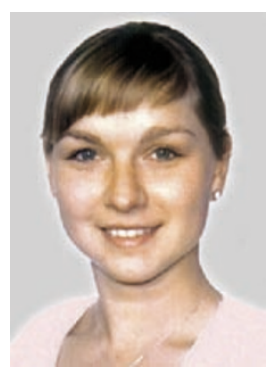

Annika Brauns hat nach ihrem Abitur in Bretten bei Karlsruhe bis 2008 an der Ruprecht-Karls Universität in Heidelberg und ein Semester an der Friedrich-Schiller-Universität in Jena Anglistik und Biologie studiert. Während ihres Studiums nahm sie eine neunmonatige Tätigkeit als Fremdsprachenassistentin in Großbritannien an. Ihre Examensarbeit schrieb Annika Brauny am Zoologischen Institut Heidelberg unter der Leitung von Prof. Thomas Braunbeck. Derzeit befindet sie sich im Referendariat am Helmholtz Gymnasium in Heidelberg. 
Der Einfluss von Globalisierung und Klimaveränderung auf die Entwicklung von Stechmücken und von ihnen übertragenen Krankheiten in Zentraleuropa

\section{Norbert Becker}

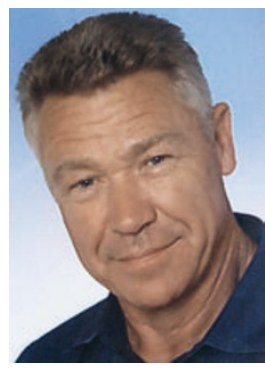

PD Dr. Norbert Becker ist seit 1976 Lehrbeauftragter am Zoologischen Institut der Universität Heidelberg und hält Kurse/Seminare in angewandter Ökologie und medizinischer Entomologie. Seit 1981 ist er hauptamtlich wissenschaflicher Direktor der KABS (German Mosquito Control Association), verantwortlich für die Bekämpfung der Stechmücken mit umweltverträglichen, biologischen Bekämpfungsstoffen. Von 1989-1994 war er Mitglied des Beraterkomitees (Steering committee for BCV) für Biologie und Bekämpfung von Vektoren (BCV) der Weltgesundheitsorganisation (WHO/TDR) in Genf. Seit 1995 ist er beratend für die WHO/TDR in Fragen der biologischen Stechmückenbekämpfung in Asien, Afrika and Südamerika tätig. Er war Direktor der „Society for Vector Ecology“ (SOVE European Region) von 1988-1990 und Präsident der weltweit aktiven SOVE im Jahr 1993. Er ist seit 1998 als Dozent am Schweizerischen Tropeninstitut im Rahmen des Health Care and Management in Tropical Countries (HCMTC) tätig. Als Ehrungen erhielt er den Distinguished Service Award der „Society for Vector Ecology“ im Jahre
1997, im Jahr 2001 die „Escherich Medaille“ von der Deutschen Gesellschaft für Entomologie für herausragende Leistung im Bereich der angewandten Biologie. 2004 wurde ihm das „Bundesverdienstkreuz am Bande“ für Verdienste um das Gemeinwohl im Rahmen der biologischen Stechmückenbekämpfung überreicht.

Aspekte zur Diversität der Polychaeta (Annelida) unter besonderer Berücksichtigung der Publikationen von Volker Storch nebst der Beschreibung einer neuen Art der Ampharetidae

\section{Thomas Wehe}

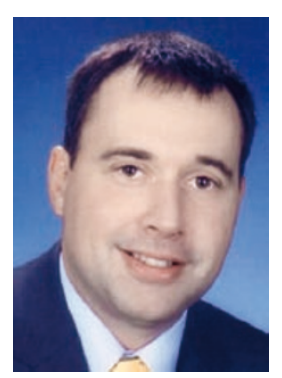

Dr. Thomas Wehe ist Diplom-Biologe. Von 2003 bis 2005 war er wissenschaftlicher Mitarbeiter am Forschungsinstitut und Naturmuseum Senckenberg, Frankfurt a. M., von 2003 bis 2008 wissenschaftlicher Mitarbeiter am Lehrstuhl für Morphologie und Ökologie (Prof. Dr. Dr. h.c. V. Storch), Universität Heidelberg. Hier beschäftigte er sich speziell mit der Biodiversität und Morphologie der $\mathrm{Po}$ lychaeta. Daneben gilt sein Interesse der Meeresbiologie, welche er auch in der Lehre vertreten hat. Seit 2008 arbeitet er im Bereich der regulatorischen Ökotoxikologie bei Dr.Knoell-Consult. 